\title{
Interband Cascade Laser Arrays for Simultaneous and Selective Analysis of C1-C5 Hydrocarbons in Petrochemical Industry
}

\author{
Julian Scheuermann, ${ }^{1}$ Pawel Kluczynski, ${ }^{2}$ Krzysztof Siembab, ${ }^{2}$ Mateusz Straszewski, ${ }^{2}$ \\ Jakub Kaczmarek, ${ }^{2}$ Robert Weih, ${ }^{1}$ Marc Fischer, ${ }^{\dagger 1}$ Johannes Koeth, ${ }^{1}$ Anne Schade, ${ }^{3}$ and \\ Sven Höfling 3,4 \\ ${ }^{1}$ nanoplus Nanosystems and Technologies GmbH, Oberer Kirschberg 4, 97218 Gerbrunn, \\ Germany \\ ${ }^{2}$ Airoptic Sp. z o.o., ul. Rubiez 46, 61-612 Poznan, Poland \\ ${ }^{3}$ Technische Physik, Physikalisches Institut and Wilhelm Conrad Röntgen Research Center \\ for Complex Material Systems, Universität Würzburg, Am Hubland, 97074 Würzburg, \\ Germany \\ ${ }^{4}$ SUPA, School of Physics and Astronomy, University of St Andrews, St Andrews, KY16 \\ 9SS, United Kingdom
}

\begin{abstract}
The detection and measurement of hydrocarbons is of high interest for a variety of applications, for example within the oil \& gas industry from extraction throughout the complete refining process, as well as for environmental monitoring and for portable safety devices. This paper presents a highly sensitive, selective and robust tunable laser analyzer that has the capability to analyze several components in a gas sample stream. More specifically, a multi-gas system for simultaneous detection of $\mathrm{C} 1$ to iC5 hydrocarbons, using a room temperature distributed feedback interband cascade laser array, emitting in the 3.3 micrometer band has been realized. It combines all the advantages of the tunable laser spectroscopy method for a fast, sensitive and selective in-line multicomponent tunable laser analyzer. Capable of continuous and milliseconds fast monitoring of $\mathrm{C} 1-\mathrm{iC} 5$ hydrocarbon compositions in a process stream, the analyzer requires no consumables (e.g. purging, carrier gas) and no in-field calibration, enabling a low cost of ownership for the analyzer.

The system was built, based on an industrial GasEye series platform and deployed for the first time in field at Preem refinery in Lysekil, Sweden in autumn 2018. Results of the measurement campaign and comparison with gas chromatography instrumentation is presented.
\end{abstract}

Keywords: gas analyzer, tunable laser, interband cascade laser, mid infrared, process control

†28. January 2019 


\section{Introduction}

Tunable laser spectroscopy (TLS) has become a powerful technique for analyzing components both in liquids ${ }^{1}$ and in gas mixtures. ${ }^{2,3}$ The major benefits of this technique are the real-time measurement speed, its high sensitivity and selectivity. ${ }^{4}$ TLS sensor systems can be built in a maintenance free fashion without the need for additional calibration or alignment after initial deployment. One of the biggest advantages is that all calibration work and the required consumables such as calibration gases are eliminated. This leads to significantly lower maintenance costs during operation (cost of ownership). The utilized wavelength range depends on the absorption of the targeted compounds. In general, the mid-infrared spectral range is of high interest since most molecular gases have their fundamental rotationalvibrational lines there. In our case, the hydrocarbons absorption bands in the 3 to $3.5 \mu \mathrm{m}$ range were chosen. Fig. 1 shows the absorption cross-section of the targeted hydrocarbons, namely methane $(\mathrm{C} 1)$, ethane $(\mathrm{C} 2)$, propane $(\mathrm{C} 3)$, butane $(\mathrm{C} 4)$, pentane $(\mathrm{C} 5)$, isobutane (iC4) and isopentane (iC5). The characteristic absorption peaks of the smaller molecules like methane and ethane are clearly visible, whereas bigger hydrocarbons form a broad absorption band, ranging over multiple hundreds of nanometers.

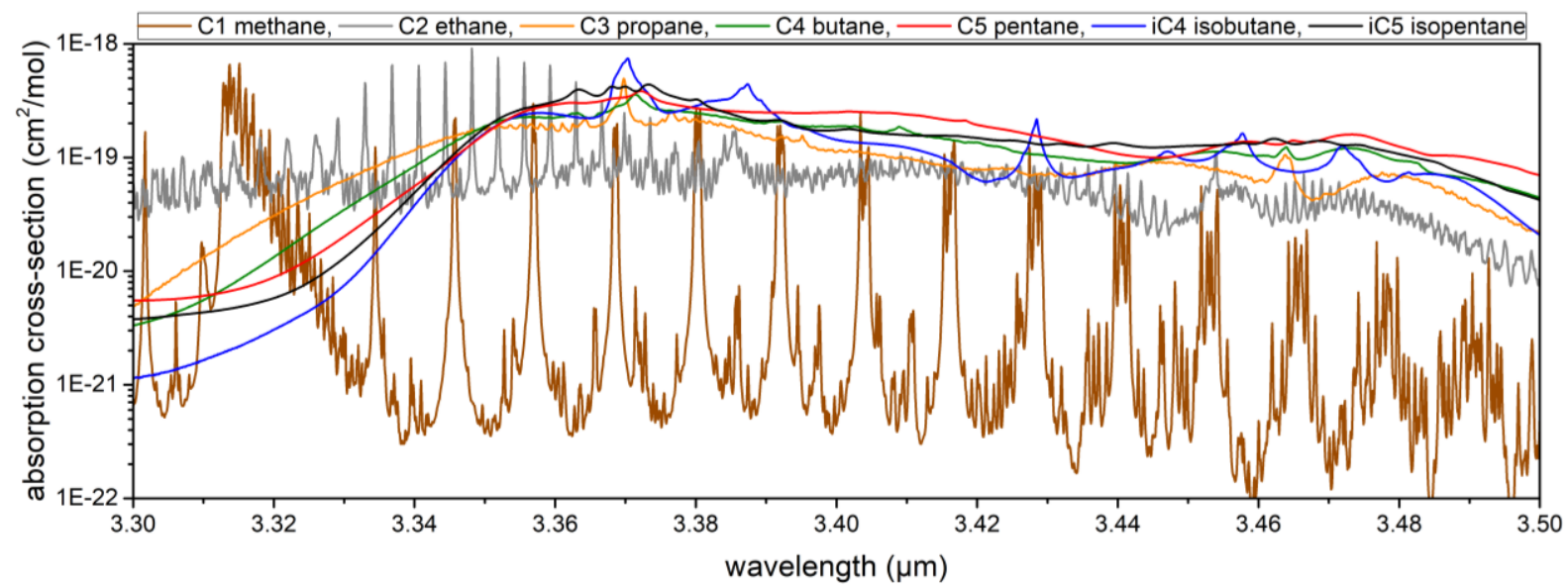

Figure 1. FTIR absorption spectra of all seven targeted hydrocarbons. The data was taken from the PNNL spectroscopic database..$^{5}$ All samples were measured individually with $1 \mathrm{ppm} \times \mathrm{m}$ at $296 \mathrm{~K}$ with a spectral resolution of $0.112 \mathrm{~cm}^{-1}$.

The interband cascade (IC) lasers technology, ${ }^{6,7}$ which is used for the light sources in this work, has its emission regime in the mid infrared region from around 3 to 7 micrometer. ${ }^{8,9}$ Various different techniques for the fabrication of spectrally single-mode devices were presented up to now. ${ }^{10-13}$ In addition, IC devices have proven their robustness ${ }^{14}$ making it possible for sensor systems to operate unattended for years. The major competing technologies are GaSb-based type-I transition diode lasers in the 3 to $4 \mu \mathrm{m}$ regime $^{15-17}$ and the quantum cascade lasers which are able to cover the whole range of interest. ${ }^{18}$ The benefits of these technologies vary depending on application requirements. In our case, we favored the IC 
technology because it yields sufficient output power and has low total power consumption, which is beneficial when integrating multiple waveguides on a single chip. In the next sections, we will describe the fabrication of the laser sources and present measurements of the targeted hydrocarbons.

\section{Laser sources}

The material we used was grown by molecular beam epitaxy on $n-G a S b$ (100) substrate in a fashion similar to ${ }^{19,20}$ and contained 6 active stages. The quantum wells were composed of $\mathrm{AlSb} / \mathrm{InAs} / \mathrm{Ga}_{0.65} \mathrm{In}_{0.35} \mathrm{Sb} / \mathrm{InAs} / \mathrm{AlSb}$ with thicknesses of 2.5/1.7/3.0/1.3/1.0 nm. The transition energy in the type-II quantum well was fine-tuned via the thicknesses of the two InAs layers to match the targeted emission wavelength. The thicknesses of the separate confinement (SCL) and cladding layers were matched for this wavelength range, in order to get good mode guidance and reasonable overlap with the active region. Therefore, the SCL layers thickness was chosen to be $320 \mathrm{~nm}$ each and the upper (lower) cladding layer to $1.7 \mu \mathrm{m}$ $(2.5 \mu \mathrm{m})$. To investigate material properties, shallowly etched broad area devices with a waveguide width of $0.1 \mathrm{~mm}$ and a length of $2 \mathrm{~mm}$ were processed. The broad area devices were measured in pulsed operation. A threshold current density of $0.29 \mathrm{kA} / \mathrm{cm}^{2}$ and a characteristic temperature of $58 \mathrm{~K}$ were determined (not shown). The single-mode devices were fabricated in a different manner: On the sample, the individual emitters were designed with a waveguide width of around $5 \mu \mathrm{m}$ and a cavity length of $0.9 \mathrm{~mm}$. The spacing between adjacent waveguides was $0.1 \mathrm{~mm}$. The waveguides were patterned using a $\mathrm{Cl} / \mathrm{Ar}$ based dry etch process, followed by reactively sputtered passivation layers consisting of silicon-nitride and silicon-oxide. A metal grating structure, defined by electron beam lithography, was deposited alongside the waveguide. The metal grating acts as distributed feedback element (DFB) ${ }^{10}$ and was designed to target the shorter wavelengths at lower operation currents and the longer wavelengths at higher operation currents. As a result, when keeping the heatsink temperature fixed, the difference in Joule heating is shifting the transition energy of the typeII quantum well and hence the spectral position of the material gain. This leads to a better alignment of gain position towards DFB emission wavelength, but requires additional parameters like thermal resistance of the chip and exact knowledge of the refractive index for successful grating design. Different sets of grating periodicities were applied to neighboring waveguides in repeating blocks of up to nine wavelengths in total. The contact metallization was sputter deposited whereas $\mathrm{AuGe} / \mathrm{Ni} / \mathrm{Au}$ was applied as a shared bottom and $\mathrm{Ti} / \mathrm{Pt} / \mathrm{Au}$ was applied as structured, electrically separated top contact (Fig. 2 left). As an example a die with a total of nine emitters per chip and geometry $(\mathrm{LxWxH})$ of $0.9 \times 1.0 \times 0.15 \mathrm{~mm}$, is presented. After facet coating with a protective layer on the front and a metal based high reflectivity coating on the back, the device was soldered on a heatspreader and mounted on a metal submount subsequently (Fig. 2 right). 

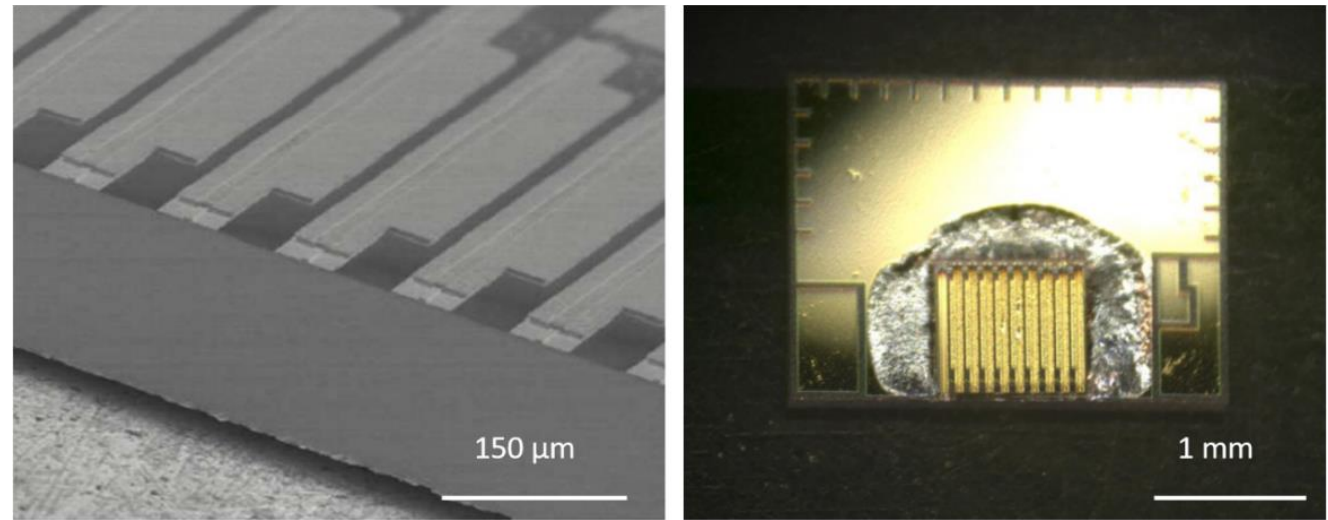

Figure 2. Electron-microscope image of a cleaved laser bar (left). The individual waveguides and the separate contact pads are visible. The right image shows an optical microscopy image of the mounted chip with nine waveguides and their individual contact pads.

For integration into the sensor system, the DFB ICL arrays were mounted into a TO66type header including a thermoelectric cooler and were hermetically sealed using a cap with an AR coated window. The setup was designed to yield two TO66-type headers, each containing an ICL array with four waveguides. The devices were temperature stabilized by the integrated thermoelectric cooler, controlled via two temperature modules and the sensor system mainboard (MB). The devices were operated in continuous wave mode at room temperature and reached output powers of around $10 \mathrm{~mW}$. Fig. 3 shows eight emission spectra, recorded from two ICL arrays. The two devices were fabricated from an identical epitaxy, and it was possible to address certain reference points in a wavelength range of $200 \mathrm{~nm}$.

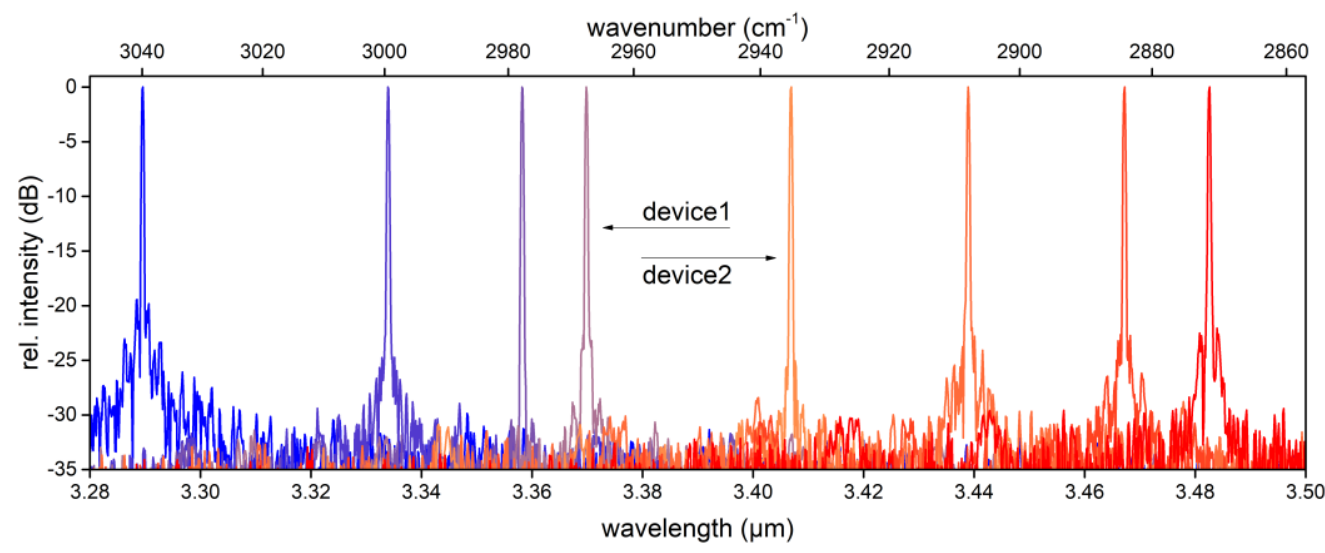

Figure 3. Single-mode emission spectra, recorded from two ICL array devices with four emitters each. Based on the same IC epitaxy, a wavelength range of $200 \mathrm{~nm}$ was addressed at certain reference points. 


\section{Experimental}

The analyzer was designed by Airoptic based on their industrial GasEye spectrometer platform and tailored specifically to incorporate laser arrays with up to eight individual channels. A schematic of the experimental setup is shown in Fig. 4. The system consists of the ICL array sources, mercury cadmium telluride (MCT) main detector, auxiliary reference detector (InAs), driver \& receiver electronics, low level signal processing means, synchronization means (on the MB) and high level signal processing means (on the HOST board). The laser sources are temperature-controlled at a specific sink temperature by a builtin Peltier via a dedicated temperature controller (temp CTRL) and stabilized within a few milli-Kelvin. The source driver, consisting of the current driver and the current multiplexer, is responsible for activation and deactivation of the individual wavelength channels. The driver is pre-programmed specifically for the $\mathrm{C} 1$ to iC5 target wavelengths, which are addressed by the individual waveguides of the ICL array. Each wavelength channel provides individual current bias and current modulation settings. A measurement sequence consists of multiplexing the current driver from emitter one to eight. For each emitter, the preprogrammed current sweep is performed. This leads to a shift in the emission wavelength in the range of a few nanometers, which was determined using a Germanium Fabry-Pérot interferometer (Ge FP). The wavelength modulated light exiting the laser source interacts with the gas matrix inside the gas cell, creating a time-dependent, intensity variation of the light impinging on the MCT detector. At the receiver end, the photocurrent is converted into a voltage signal, filtered and amplified in the analog domain using a bandpass filter and then subsequently digitized by a high-speed A/D converter. The raw spectrum is being further processed in the digital domain, using a digital demodulation filter and normalization, before it is sent to the $\mathrm{MB}$ for numerical analysis in order to extract the gas concentration information from the optical spectra. The procedure can be referred to as a wavelength modulation spectroscopy (WMS) approach, which is performed sequentially for the eight different wavelength channels. In our case the artificial neural network (ANN) does the evaluation of the obtained measurement. The auxiliary InAs reference detector is used for frequency and amplitude stabilization of the source as well as enhancement of system performance by suppressing common mode noise. Within the receiver, the signal is demultiplexed and demodulated. The MB unit controls system-level supervisory functions such as multiplexing control and timing synchronization between the driver and receiver. 


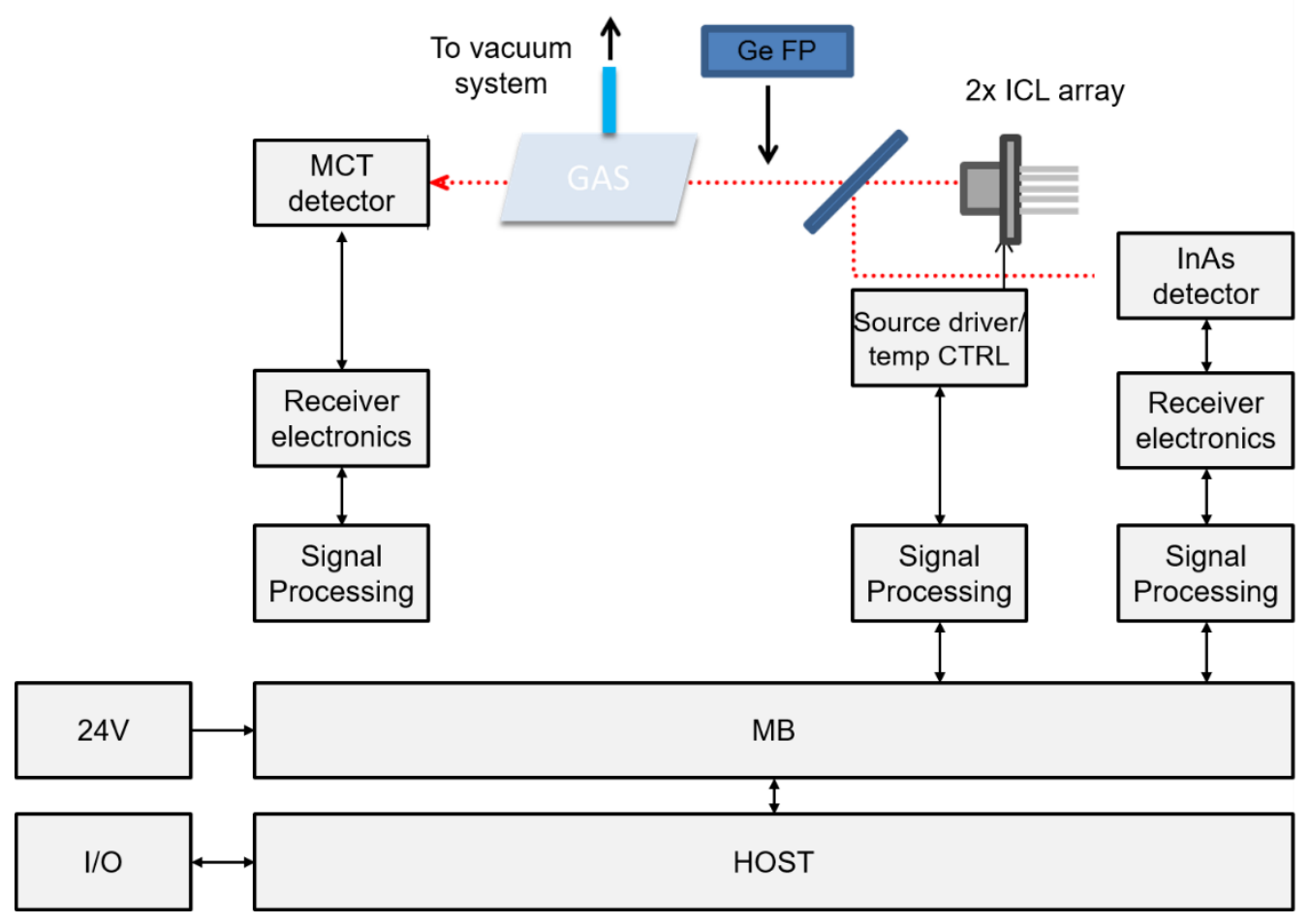

Figure 4. Schematics of the analyzer

In the analyzer, we used two TO66 cans with four DFBs emitters in each array chip. The laser driver provided drive currents to each individual DFB in the array in a certain sequence, which is stored in a look-up table. Each of the arrays were temperature stabilized using two independent temperature controllers. The laser beams from two independent TO66 cans were combined by a beam combiner and collimated using a specially designed $\mathrm{ZnSe}$ lens. The beam was transmitted through a $2 \mathrm{~mm}$ long gas cell and focused on the cooled MCT detector. The optics were designed accordingly to allow the eight beams to fall on the detector's active area $(1 \mathrm{~mm}$ diameter). This was possible due to the relatively short path length of the spectrometer, which also allowed the analyzer to fit in a standard 4U 19 inch rack type enclosure, see Fig. 5 (right). The short path length of the cell was chosen to be sufficient, since the application is targeting relatively high concentrations of the respective analytes. With an integration time of three seconds, the detection limit of the system is at $10 \mathrm{ppb}^{*} \mathrm{~m}$ for $\mathrm{C} 1$ to $\mathrm{C} 3$, at $20 \mathrm{ppb}^{* \mathrm{~m}}$ for $\mathrm{C} 4 \& \mathrm{iC} 4$ and at $50 \mathrm{ppb} * \mathrm{~m}$ for $\mathrm{C} 5 \& \mathrm{iC} 5$, respectively. The detection limit could be further decreased by using longer integration time or by adding a second gas cell with longer path length. The system has a maximum integration time of $120 \mathrm{~s}$ after which further integration will not improve limit of detection. Depending on the integration time, the system has a dynamic range of around $10^{6}$. This is well comparable with the dynamic range of gas chromatographs, which are in the range of $10^{3}$ to $10^{7}$, depending on the used detector. The main advantage of the laser based sensor system is low maintenance, 
since it needs no recalibration and it can automatically compensate for transmission loss due to for example fouling of the windows in harsh environments.

\section{Calibration process using neural network}

The instrument was calibrated with a gas mixing station which was specially prepared for this purpose The $\mathrm{C} 1-\mathrm{iC} 4$ gases were mixed in the gas dividers using 100vol\% gas cylinders whereas the $\mathrm{C} 5$ and iC5 calibration gases were prepared in nominal concentrations of $5 \mathrm{vol} \%$ $\mathrm{C} 5$ and $5 \mathrm{vol} \%$ iC5 in $\mathrm{N}_{2}$ buffer. The calibration process involved the preparation of 20 different mixtures for each gas, ranging from $0-100 \mathrm{vol} \%$ in $5 \mathrm{vol} \%$ steps for $\mathrm{C} 1-\mathrm{iC} 4$, whereas iC5 and C5 were calibrated for a range of $0-5 \mathrm{vol} \%$ using $0.25 \mathrm{vol} \%$ steps. This process was done semi-automatic. Additionally, approximately 2500 different spectral mixtures, created by digital superposition, were included. The resulting spectra were used as input for the ANN. Each spectral vector contained a maximum of 24 channels corresponding to 8 lasers $\mathrm{x} 3$ segments, each segment containing 128-long spectral points. After the data acquisition had been completed, the spectral information was fed into a dedicated program which calculated the neural network's weights and biases. After obtaining a set of parameters, they were uploaded onto the analyzer firmware. A set of ANNs, each with one hidden layer and backpropagation, was used to compute the ppm concentration of the individual gases in a mixture. The reason for this approach was to avoid over learning when using a larger and more complex ANN. The learning procedure and the used type of network were adjusted accordingly to minimize interferences with other gases. More details about the analyzer and calibration method have been presented in Ref. 21. Moreover, all expected gas species and their estimated concentrations were defined together with Preem prior to the learning procedure. In the presence of gases with spectral features of similar magnitude you have to include them in the learning process. In this specific case, the system has an automatic detection of excess $\mathrm{H}_{2} \mathrm{O}$ and $\mathrm{CO}_{2}$ in the process line.

\section{Results and Discussion}

The analyzer was installed on the site at Preem $^{22}$ in September 2018. The analyzer was placed inside the analyzer house at the 2503 Propane Tower. The connections were prepared by Preem refinery in advance prior to arrival of the Airoptic instrument, which allowed for a quick and swift commissioning in just a couple of hours from the arrival of the analyzer. Due to a small form factor of the analyzer, handling was quite easy and did not require special equipment or tools to install it in an existing analyzer house (see Fig. 5 top images). All electrical as well as gas connections to the analyzer are located in the rear of the enclosure and are clearly marked. A photo of the rear panel is shown in Fig. 5, bottom image. The sample line was shared with a Siemens MicroSAM gas chromatograph (GC), installed in a shelter back-to-back with the analyzer house. The connection was fed through a dedicated opening in the back of the analyzer house, see Fig. 6. 

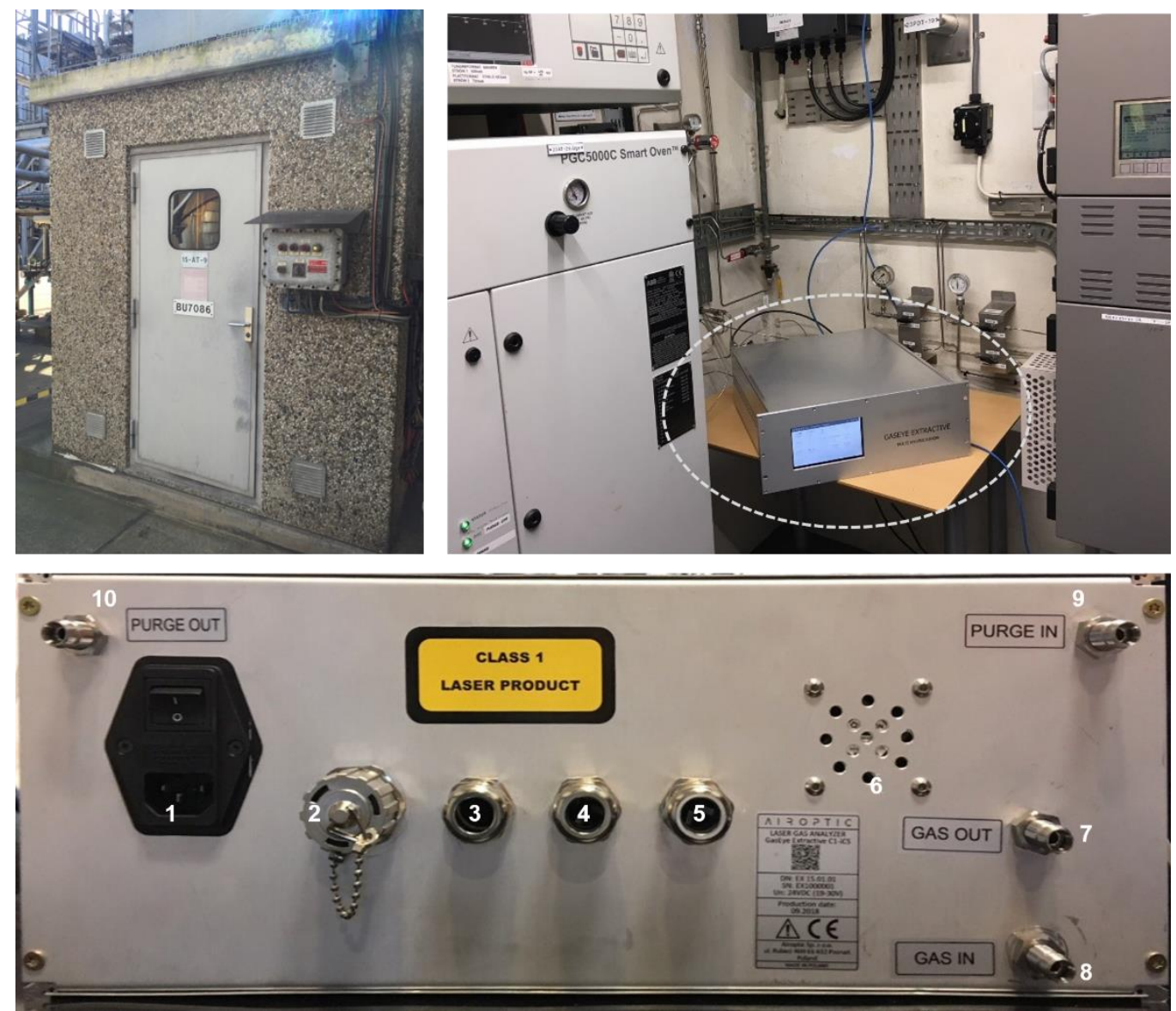

Figure 5. The sensor system in the analyzer house at Preem refinery (top images). The overview of connection ports on the rear panel (bottom image).

The corresponding numbers refer to: (1) power inlet $230 \mathrm{VAC} 50 \mathrm{~Hz}$, (2) service ethernet port RJ-45, (3),(4),(5) I/O cable glands, (6) ventilation outlet, (7),(8) sample gas inlet/outlet, (9),(10) purge inlet/outlet.

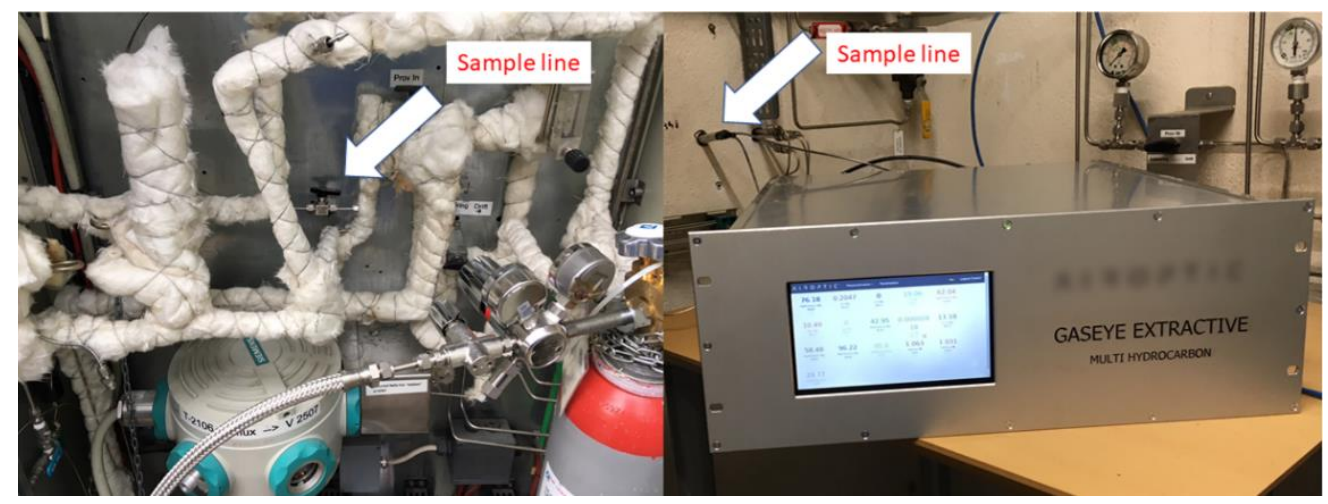

Figure 6. Sample line from shelter to the analyzer 
The system was factory calibrated and required no calibration on-site. The analyzer was set to an integration time of three seconds, was equipped with Modbus TCP/IP and 4-20 mA analog output interface for continuous data transfer to the distributed control system of the refinery. The ethernet connection to the control room was established after the analyzer was connected to gas lines. It was possible to access both webserver application as well as the GasEye Viewer logger program from the Preem control room via a dedicated IP address within Preem's network. It was also possible to access the control room computer from the Airoptic facility via VPN channel at designated hours. The viewer program for data acquisition was running for $24 / 7$ and the data was logged every five seconds into the database. As example, Fig. 7 shows C2 to iC4 measurements of a Siemens MicroSAM GC and the Airoptic system in comparison.
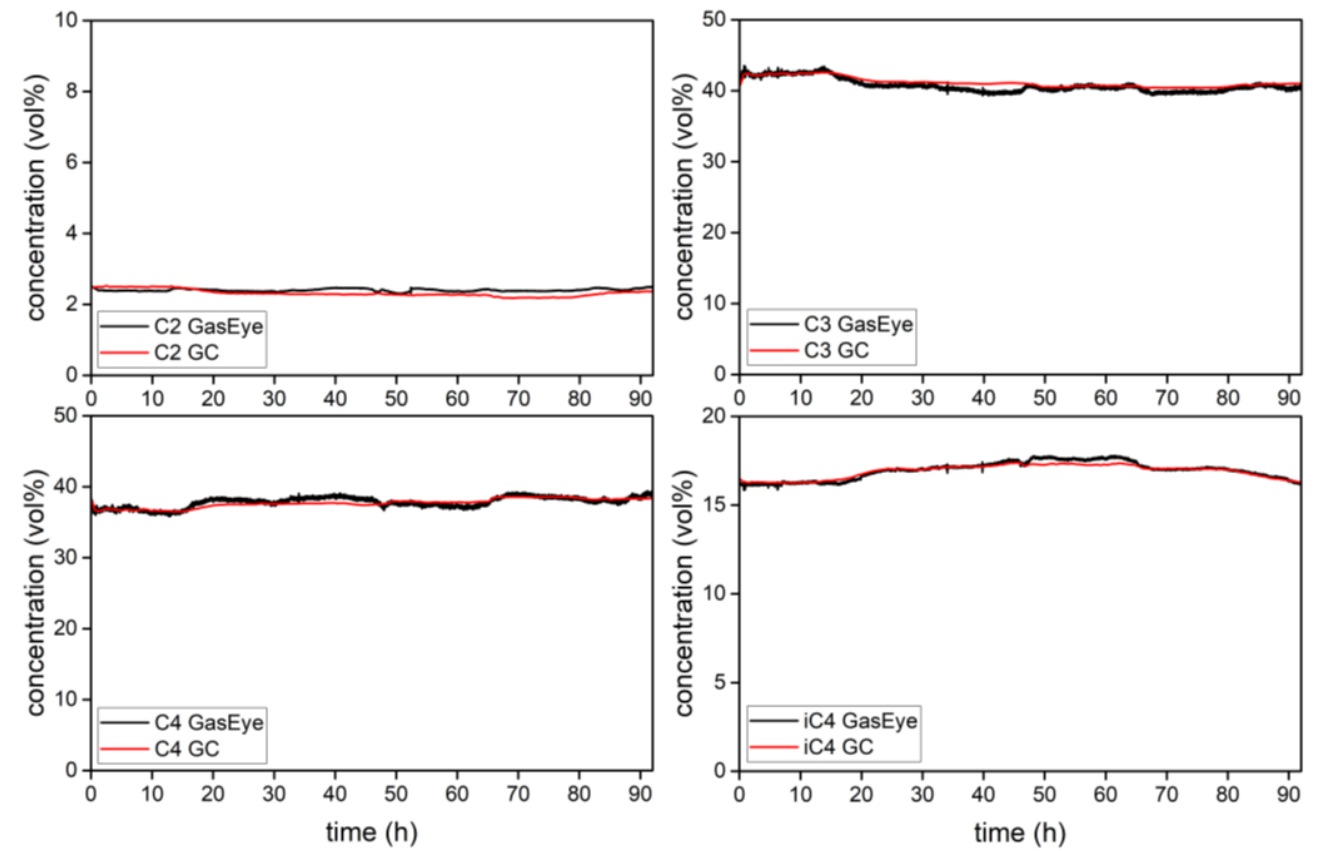

Figure 7. The graph depicts examples of the time-dependent concentration of ethane, propane, butane and isobutane in the gas matrix taken from the sample line at the analyzer house. The 90 hour logs show the comparison of a GC and the presented sensor system.

As we can see the agreement between the GC and the tunable lasers is good and well within the accuracy limit ( $2 \%$ of the read value) of the analyzer. Due to the much faster response time of the GasEye analyzer the system was able to detect any rapid changes in the gas concentration which normally are not observed in a GC signal. Generally, the TLS sensor system is complementing the properties of other sensor systems. Especially when working with a known gas matrix, like in industrial process control, a TLS system can be fine-tuned to match the required specifications of e.g. response time, detection limit etc. When working in 
undefined environments or when interference with unexpected gas species cannot be avoided, GCs for example may be better suited.

\section{Conclusion}

In this paper, we have demonstrated the simultaneous measurement of methane, ethane, propane, butane, pentane, isobutane and isopentane using TLS with two single-mode interband cascade laser arrays and a neural network based evaluation scheme. The sensor system has been deployed in field and was running 24/7 for months. The results are in good agreement with other detector platforms, located at the same gas extraction point. The extensive device characterization showed that the underlying ICL material is capable of addressing the targeted $200 \mathrm{~nm}$ wavelength range with a single laser array operated at a fixed temperature. Additionally, the flexibility of such a laser array could be further increased by incorporating waveguides with extended wavelength tuning capability. ${ }^{23}$

\section{Acknowledgements}

This research was supported by Preem AB who assisted with their expertise throughout the development phase and made it possible to deploy the system in field in one of their refineries.

\section{Funding}

This project has received funding from the European Union's Horizon 2020 research and innovation programme under grant agreement No 636930 (iCspec).

\section{References}

1. J. Kuligowski, G. Quintás, B. Lendl. "High Performance Liquid Chromatography with On-Line Dual Quantum Cascade Laser Detection for the Determination of Carbohydrates, Alcohols and Organic Acids in Wine and Grape Juice". Appl. Phys. B. 2010. 99(4): 833840. doi:10.1007/s00340-010-4013-y.

2. S. Lundqvist, P. Kluczynski, R. Weih, et al. "Sensing of Formaldehyde Using a Distributed Feedback Interband Cascade Laser Emitting around 3493 nm". Appl. Opt. 2012. 51(25): 6009-6013. doi:10.1364/AO.51.006009.

3. R. Heinrich, A. Popescu, R. Strzoda, et al. "High Resolution Quantitative Multi-Species Hydrocarbon Gas Sensing with a cw External Cavity Quantum cascade Laser Based Spectrometer in the 6-11 $\mu \mathrm{m}$ Range". J. Appl. Phys. 2019. 125(13): 134501. doi:10.1063/1.5082168.

4. L. Dong, F.K. Tittel, C. Li, et al. "Compact TDLAS Based Sensor Design Using Interband Cascade Lasers for Mid-IR Trace Gas Sensing". Opt. Express. 2016. 24(6): A528-A535. doi:10.1364/OE.24.00A528. 
5. S.W. Sharpe, T.J. Johnson, R.L. Sams, et al. "Gas-Phase Databases for Quantitative Infrared Spectroscopy". Appl. Spectrosc. 2004. 58(12): 1452-1461. doi:10.1366/0003702042641281.

6. R.Q. Yang. "Infrared Laser Based on Intersubband Transitions in Quantum Wells". Superlattices Microstruct. 1995. 17(1): 77-83. doi:10.1006/spmi.1995.1017.

7. I. Vurgaftman, R. Weih, M. Kamp, et al. "Interband Cascade Lasers". J. Phys. D: Appl. Phys. 2015. 48(12): 123001. doi:10.1088/0022-3727/48/12/123001tt.

8. J. Scheuermann, R. Weih, M. von Edlinger, et al. "Single-Mode Interband Cascade Lasers Emitting Below 2.8 m". Appl. Phys. Lett. 2015. 106(16): 161103. doi:10.1063/1.4918985.

9. M. Dallner, F. Hau, S. Höfling, et al. "InAs-Based Interband-Cascade-Lasers Emitting around $7 \mu \mathrm{m}$ with Threshold Current Densities Below $1 \mathrm{kA} / \mathrm{cm}^{2}$ at Room Temperature". Appl. Phys. Lett. 2015. 106(4): 041108. doi:10.1063/1.4907002.

10. R. Weih, L. Nähle, S. Höfling, et al. "Single Mode Interband Cascade Lasers Based on Lateral Metal Gratings". Appl. Phys. Lett. 2014. 105(7): 071111. doi:10.1063/1.4893788.

11. C.S. Kim, M. Kim, J. Abell, et al. "Mid-Infrared Distributed-Feedback Interband Cascade Lasers with Continuous-Wave Single-Mode Emission to 80" $"$. Appl. Phys. Lett. 2012. 101(6): 061104. doi:10.1063/1.4744445.

12. M. von Edlinger, J. Scheuermann, R. Weih, et al. "Monomode Interband Cascade Lasers at $5.2 \mu \mathrm{m}$ for Nitric Oxide Sensing". IEEE Photonics Technol. Lett. 2014. 26(5): 480-482. doi:10.1109/LPT.2013.2297447.

13. F. Xie, M. Stocker, J. Pham, et al. "Distributed Feedback Interband Cascade Lasers with Top Grating and Corrugated Sidewalls". Appl. Phys. Lett. 2018. 112(13): 131102. doi:10.1063/1.5009741.

14. S. Forouhar, C. Borgentun, C. Frez, et al. "Reliable Mid-Infrared Laterally-Coupled Distributed-Feedback Interband Cascade Lasers". Appl. Phys. Lett. 2014 105(5): 051110. doi:10.1063/1.4892655.

15. A. Bauer, K. Rößner, T. Lehnhardt, et al. "Mid-Infrared Semiconductor Heterostructure Lasers for Gas Sensing Applications". Semicond. Sci. Technol. 2010. 26(1): 014032. doi:10.1088/0268-1242/26/1/014032.

16. L. Shterengas, G. Kipshidze, T. Hosoda, et al. "Cascade Pumping of 1.9-3.3 $\mu \mathrm{m}$ Type-I Quantum Well GaSb-Based Diode Lasers". IEEE J. Sel. Top. Quantum Electron. 2017. 23(6): 1-8 doi:10.1109/JSTQE.2017.2687763.

17. K. Vizbaras, A. Vizbaras, A. Andrejew, et al. "Room-Temperature Type-I GaSb-Based Lasers in the 3.0-3.7 $\mu \mathrm{m}$ Wavelength Range". Proc. SPIE 8277, Novel In-Plane Semiconductor Lasers XI. 2012. 8277: 82771B. doi:10.1117/12.905930.

18. M.S. Vitiello, G. Scalari, B. Williams, et al. "Quantum Cascade Lasers: 20 Years of Challenges". Opt. Express. 2015. 23(4): 5167-5182. doi:10.1364/OE.23.005167.

19. I. Vurgaftman, W.W. Bewley, C.L. Canedy, et al. "Rebalancing of Internally Generated Carriers for Mid-Infrared Interband Cascade Lasers with Very Low Power Consumption". Nat. Commun. 2011. 2: 585. doi:10.1038/ncomms1595. 
20. R. Weih, M. Kamp, S. Höfling. "Interband Cascade Lasers with Room Temperature Threshold Current Densities Below 100 A/cm²". Appl. Phys. Lett. 2013. 102(23): 231123. doi:10.1063/1.4811133.

21. P. Kluczynski, K. Siembab, J. Derezynski, et al. "Multi-Component Tunable Laser Analyzer for Process Control". presented at: ISA Analysis Division Symposium, Pasadena, California; April 23-27, 2017.

22. Preem AB. "About Preem - Preem.se". 2019. https://www.preem.se/en/in-english/about/ [accessed Oct 28 2020].

23. M. von Edlinger, R. Weih, J. Scheuermann, et al. "Monolithic Single Mode Interband Cascade Lasers with Wide Wavelength Tunability". Appl. Phys. Lett. 2016. 109(20): 201109. doi:10.1063/1.4968535. 